\title{
PREVALÊNCIA DE DESNUTRIÇÃO E CONSUMO ALIMENTAR EM ESCOLARES DE UMA ESCOLA MUNICIPAL DE JOINVILLE-SC
}

\author{
PREVALENCE OF MALNUTRITION IN SCHOOL CHILDREN OF A MUNICIPAL SCHOOL IN \\ JOINVILLE-SC
}

\author{
Jaqueline Emily Chagas ${ }^{{ }^{*}}$ \\ Orcid: https://orcid.org/0000-0002-6444-2882
}

\author{
Camila Cristina Debortoli ${ }^{b^{*}}$ \\ Orcid: https://orcid.org/0000-0001-7630-1158
}

jaquelineemilychagas@gmail.com ${ }^{\mathrm{a}}$, camila.debortoli@ielusc.br ${ }^{\mathrm{b}}$ Associação Educacional Luterana Bom Jesus/IELUSC*

Data de Submissão :23/05/2019

Data de Aceite: 14/11/2019

\section{RESUMO}

Introdução: A desnutrição infantil ainda é um problema que acomete várias regiões do Brasil. Em localidades mais desenvolvidas ainda há casos de crianças, em idade escolar, em vulnerabilidade social e insegurança alimentar. Objetivo: Investigar a prevalência de desnutrição e o comportamento alimentar em estudantes de uma escola municipal na cidade de Joinville/SC. Materiais e métodos: A pesquisa abrangeu crianças de 7 a 10 anos de idade, de ambos os sexos, matriculadas em uma escola municipal no período matutino. Os dados coletados foram peso, estatura, idade e para classificação do estado nutricional foram aplicadas as curvas de crescimento da OMS do ano de 2007, de IMC para a idade, peso para a idade e estatura para a idade e para a análise do consumo alimentar o questionário QUADA. Resultados: Participaram da pesquisa 101 crianças, com média de idade de 8,52 sendo 58,4\% do sexo feminino e 41,6\% do sexo masculino. Dentre estas, $1 \%$ apresentou IMC abaixo para idade, $5 \%$ peso abaixo para a idade e $3 \%$ estatura inadequada para a idade. Em relação ao consumo alimentar, o maior percentual de adequação conforme as porções recomendadas pela Pirâmide Alimentar Infantil Brasileira, esteve no grupo de carnes e ovos atingindo 56,4\%. Conclusão: Existem casos de desnutrição em grandes centros urbanos, em quantidade diminuída. Estudos como este, demonstram grande importância para o reconhecimento da transição nutricional, visualizando a classificação nutricional e a qualidade dos alimentos consumidos pelas crianças nos dias de hoje.

Palavras-chave: Desnutrição proteico-calórica; consumo de alimentos; estudantes

\begin{abstract}
Introduction: Child malnutrition is still a problem that affects several regions of Brazil. In more developed localities there are still cases of school-aged children in social vulnerability and food insecurity. Objective: To investigate the prevalence of malnutrition and eating behavior in students of a municipal school in the city of Joinville / SC. Materials and methods: The study covered children from 7 to 10 years of age, of both sexes, enrolled in a municipal school in the morning. The data collected were weight, height, age and for classification of nutritional status were applied the WHO growth curves for 2007, from BMI to age, weight for age and height for age and for analysis of food consumption the QUADA questionnaire. Results: 101 children participated, with a mean age of $8.52,58.4 \%$ female and $41.6 \%$ male. Among these, $1 \%$ presented BMI below for age, $5 \%$ weight below for age and 3\% for height inadequate for age. Regarding food consumption, the highest percentage of adequacy according to the portions recommended by the Brazilian Child Food Pyramid, was in the group of meat and eggs reaching 56.4\%. Conclusion: There are cases of malnutrition in large urban centers, in diminished numbers. Studies such as this, show great importance for the recognition of the nutritional transition, visualizing the nutritional classification and the quality of the foods consumed by the children today.
\end{abstract}

Key words: Protein-caloric malnutrition; food consumption; students 


\section{Introdução}

A desnutrição infantil ainda é um problema que acomete várias regiões do Brasil, principalmente zonas mais carentes do país como no caso da região norte e nordeste. Porém, mesmo em localidades mais desenvolvidas ainda há casos de crianças em idade escolar em vulnerabilidade social e insegurança alimentar, destacando-se casos de desnutrição ${ }^{1}$.

Atualmente, evidencia-se o crescimento do sobrepeso e da obesidade infantil, com base na alimentação industrializada e desequilibrada que chega à mesa das crianças, favorecendo esta condição patológica. Fatores como alimentação inadequada, avanço tecnológico, falta da prática de atividade física, publicidade infantil, autonomia reduzida dos pais perante a alimentação dos filhos, entre outros fatores, levaram ao agravo deste quadro e consequentemente à situação atual do Brasil ${ }^{2}$.

A pobreza é uma das grandes causas de desnutrição, em que a população atingida se encontra desprovida de recursos financeiros suficientes para suprir as necessidades energéticas de sua família e proporcionar alimento na quantidade e qualidade adequada para manutenção da vida. A penúria, infelizmente, é uma realidade ainda identificada em muitas regiões do país, decorrente de fatores econômicos, demográficos, culturais, políticos e até mesmo devido à desigualdade social ${ }^{3}$.

$\mathrm{Na}$ infância, é necessário um aporte energético adequado, bem como a presença de macro e micronutrientes para proporcionar um crescimento normal, evitar deficiências no desenvolvimento cognitivo e intelectual e disfunções no organismo, que podem ser levadas para toda a vida. Nessa fase de desenvolvimento, a desnutrição energético-proteica não deve estar presente, já que uma vez o organismo debilitado, sua imunidade fica comprometida e a instalação de doenças indesejáveis se torna mais propícia ${ }^{4}$.

A insegurança alimentar e nutricional é um quadro que atinge muitas pessoas no país e no mundo, contudo as crianças. De acordo com a Lei Orgânica de Segurança Alimentar e Nutricional
(Losan) (Lei no 11.346, de 15 de julho de 2006), Segurança Alimentar e Nutricional (SAN) é definida como

a realização do direito de todos ao acesso regular e permanente a alimentos de qualidade, em quantidade suficiente, sem comprometer o acesso a outras necessidades essenciais, tendo como base práticas alimentares promotoras de saúde, que respeitem a diversidade cultural e que sejam social, econômica e mbientalmente sustentáveis ${ }^{5}$.

Dessa forma, existem no Brasil, diversos programas e políticas que visam melhorar essa situação no país, por meio de estratégias diferentes com objetivo de promover segurança alimentar e nutricional para a população brasileira. No ano de 2003 essas ações se tornaram um conjunto de políticas do programa Fome Zero, ganhando cada vez mais estímulo. Assim, foi criado o Conselho Nacional de Alimentação e Nutrição (CONSEA), sendo um órgão de suporte à Presidência da República que engloba o Sistema Nacional de Segurança Alimentar e Nutricional (SISAN), a fim de monitorar as políticas públicas de alimentação e nutrição ${ }^{6,7}$.

A avaliação nutricional é o melhor parâmetro para atenção das condições nutricionais na população infantil, visto que através dela é possível detectar a história pregressa e atual, realizar anamnese alimentar ou recordatório de 24 horas, avaliar o paciente conforme exame físico, analisar exames laboratoriais quando necessário, detectar as medidas corporais através da antropometria devidamente aplicada, determinar as necessidades nutricionais de acordo com cada criança, e ainda intervir através da educação nutricional ${ }^{8}$.

Todavia, ressalta-se a importância de estudos que contribuam na averiguação de casos de desnutrição infantil, colaborando no combate desse agravo nutricional e trazendo mais segurança alimentar à população brasileira ${ }^{9}$.

Diante do exposto, esta pesquisa tem como objetivo investigar a prevalência de desnutrição e o comportamento alimentar em estudantes de uma escola municipal na cidade de Joinville/SC. 


\section{Metodologia}

Trata-se de uma pesquisa de caráter transversal e descritiva, realizada em uma escola da rede municipal da cidade de Joinville/SC no bairro Aventureiro, na localidade do Parque Joinville. Foram incluídas no estudo todas as crianças que estavam devidamente matriculadas no período matutino, com idade escolar entre 7 e 10 anos, devidamente autorizadas por seus responsáveis e que estiveram presentes nos dias da coleta de dados. Foram excluídas crianças com necessidades específicas que não possuíam parâmetros para classificação antropométrica, bem como as que desistiram no ínterim da coleta de dados ${ }^{11,12}$. Os dados foram coletados pela acadêmica pesquisadora e três acadêmicas voluntárias do curso de nutrição, do dia 02 a 17 de outubro de 2018.

A abordagem das crianças foi feita durante as aulas de educação física, artes ou em horário de atividade extra, não prejudicando o andamento do plano de aula. Antes da coleta antropométrica, foi perguntado à criança o seu nome, a sua data de nascimento ou idade e sua sala, ambos esses dados foram anotados em planilha juntamente com o sexo da criança. Para a avaliação antropométrica foram coletados peso corporal $(\mathrm{kg})$ através de balança digital da marca DLK Sports ${ }^{\circledR}$ com capacidade máxima de $180 \mathrm{~kg}$, onde foi orientado que as crianças estivessem com vestimenta leve ou roupa de ginástica e descalças, a balança estava ligada antes da criança se posicionar no centro da mesma, ereta, com os pés juntos e os braços estendidos ao longo do corpo. Para obtenção da estatura $(\mathrm{cm})$ foi utilizado estadiômetro portátil da marca CESCORF ${ }^{\oplus}$, sendo aferida com as crianças de pé, eretas, com as pernas e pés paralelos, peso distribuído em ambos os pés, braços estendidos ao lado do corpo e palmas das mãos voltadas para a superfície vertical do estadiômetro, com a cabeça erguida, em ângulo reto com o pescoço e olhando para um ponto fixo na altura dos olhos ${ }^{13}$.

Após coleta antropométrica, todas as crianças passaram por uma anamnese alimentar individual, em que com o auxílio do Questionário Alimentar do dia Anterior - QUADA- as crianças disseram sobre sua alimentação, compreendendo café da manhã, lanche da manhã, almoço, lanche da tarde, jantar e lanche da noite. Além disso, foi perguntado qual o meio de transporte utilizado para chegar até a escola, também com auxílio do QUADA. Todos os dados foram anotados à mão pelo pesquisador, com identificação da criança por nome, $\mathrm{n}^{\circ}$ e série antes da sua anamnese.

O Índice de Massa Corporal (IMC) foi calculado pela fórmula: IMC $=$ Peso $(\mathrm{kg}) /$ Altura $^{2}(\mathrm{~m})$. E o estado nutricional foi avaliado e classificado com base nas curvas de peso por idade, estatura por idade e IMC por idade ${ }^{15}$. Os dados foram tabulados em banco de dados e analisados através de estatística descritiva no programa Microsoft Office Excel ${ }^{\circledR}$ 2010. Para análise da anamnese alimentar utilizou-se como parâmetro comparativo a pirâmide alimentar brasileira da Sociedade Brasileira de Pediatria ${ }^{16 .}$

A presente pesquisa foi aprovada pelo Comitê de Ética em Pesquisa (CEP) do BOM JESUS/ IELUSC, conforme parecer número 2.908.847, e também autorizada pela Secretaria Municipal da Educação.

\section{Resultados}

A escola em estudo possuía 204 crianças matriculadas do $1^{\circ}$ ao $5^{\circ}$ ano no período matutino, a pesquisa abrangeu 101 (49,5\%). A média de idade das crianças foi de 8,52 anos, o peso médio foi de $32,44 \mathrm{~kg}$, tendo como valores mínimos 18,5 $\mathrm{kg}$ e máximo $87,1 \mathrm{Kg}$. A média do IMC foi 18,89 $\mathrm{kg} / \mathrm{m}^{2}$, conforme apresenta a Tabela 01 . 
Tabela 01 - Médias, medianas, desvios-padrão, valores mínimos e máximos da idade, peso, estatura e IMC dos escolares avaliados.

\begin{tabular}{lccccc}
\hline & Média & Mediana & Desvio Padrão & Valor mínimo & Valor máximo \\
\hline Idade $($ anos) & 8,52 & 9 & 1,19 & 7 & 10 \\
Peso $(\mathrm{kg})$ & 32,44 & 29,8 & 10,42 & 18,5 & 87,1 \\
Estatura $(\mathrm{m})$ & 1,33 & 1,32 & 0,11 & 1,14 & 1,65 \\
IMC $\left(\mathrm{kg} \mathrm{m}^{2}\right)$ & 18,89 & 16.96 & 3,25 & 13,10 & 32,60 \\
\hline
\end{tabular}

Com relação ao sexo das crianças, houve prevalência do sexo feminino, $59(58,4 \%)$ sobre o sexo masculino, 42 (41,6\%), Tabela 02.

A escola em estudo possuía no horário matutino 71 alunos matriculados em 3 turmas de primeiros anos, 28 estudantes em uma turma de segundo ano, 24 em uma turma de terceiro ano, 54 em duas turmas de quarto ano e 33 crianças em um único quinto ano. Na pesquisa participaram 26 crianças de $1^{\circ}$ ano (25,74\%), 14 de $2^{\circ}$ ano (14,86\%), 18 de $3^{\circ}$ ano (17,82\%), 32 de $4^{\circ}$ ano (31,68\%) e 11 alunos de $5^{\circ}$ ano (10,89\%). Entre os estudantes 30 tinham 7 anos de idade (29,7\%), 16, 8 anos (15,8\%), 27, 9 anos $(26,7 \%)$ e 28 crianças tinham 10 anos $(27,7 \%)$, também exposto na Tabela 02.

Tabela 02 - Caracterização da população estudada

\begin{tabular}{lll}
\hline Variáveis & $\%$ & $\mathbf{N}$ \\
\hline Sexo & & 42 \\
Masculino & 41,6 & 59 \\
Feminino & 58,4 & 30 \\
\hline Idade & & 16 \\
7 & 29,7 & 27 \\
8 & 15,8 & 28 \\
9 & 26,7 & 26 \\
10 & 27,7 & 14 \\
\hline Ano escolar & & 18 \\
$1^{\circ}$ ano & 25,74 & 32 \\
$2^{\circ}$ ano & 14,86 & 11 \\
$3^{\circ}$ ano & 17,82 & 31,68 \\
$4^{\circ}$ ano & 10,89 & \\
$5^{\circ}$ ano & & \\
\hline
\end{tabular}

Conforme a classificação do estado nutricional segundo IMC para idade (IMC/I), uma criança obteve peso abaixo do esperado (1\%). O estado de eutrofia foi encontrado em 64 crianças (63,4\%), 16 crianças com sobrepeso representando $15,8 \%$ da amostra, e 20 obesos (19,8\%), descrito na Figura 01 . 
Figura 01 - Classificação do estado nutricional conforme a curva de IMC/Idade, OMS 2007.

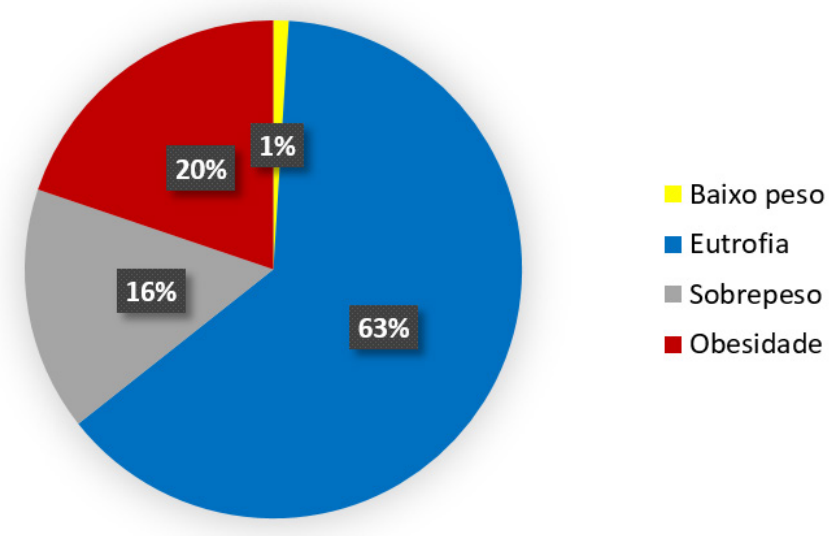

A Figura 02 apresenta os resultados da classificação do estado nutricional mediante curva de Peso para Idade (Peso/I), no qual entre os alunos, $78(77,2 \%)$ estavam com o peso adequado para idade, $18(17,8 \%)$ com peso elevado para idade e $5(5 \%)$ com baixo peso para idade. Já na Figura 03, que leva em consideração a curva de Estatura para Idade (Estatura/I), foi possível verificar que 97 (97\%) crianças assistidas estavam com a altura condizente para sua idade. Assim, apenas $3(3 \%)$ estavam com a estatura abaixo do esperado.

Figura 02 - Classificação do estado nutricional conforme a curva de Peso/Idade, OMS 2007.

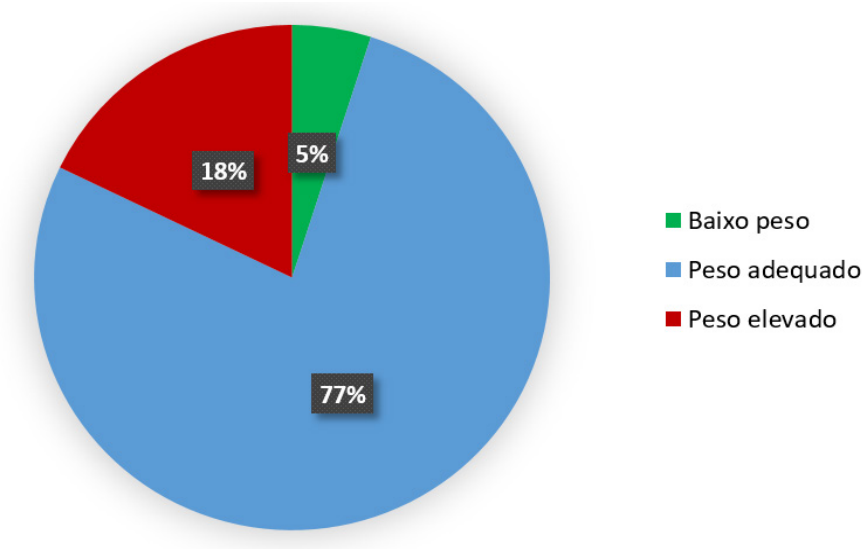


Figura 03 - Classificação da população assistida conforme Estatura para Idade, OMS 2007.

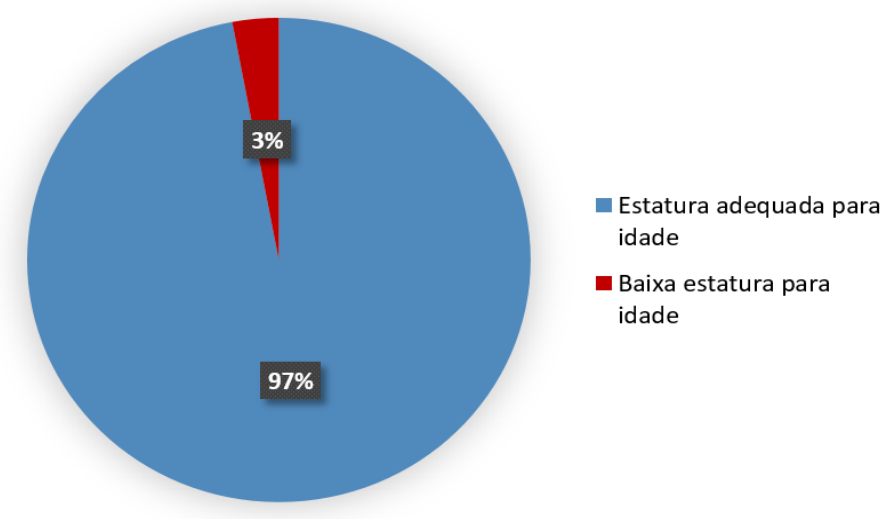

As crianças que foram classificadas com baixo do estado nutricional ${ }^{15}$, estão relacionadas na IMC/I, baixo P/I e baixa E/I, segundo classificação Tabela 03.

Tabela 03 - Relação entre as crianças com baixo IMC/I, baixo P/I e baixa E/I

\begin{tabular}{cccc}
\hline Crianças & Baixo IMC/I & Baixo Peso/I & Baixa Estatura/I \\
\hline A & Sim & Sim & Não \\
B & Não & Sim & Não \\
C & Não & Sim & Não \\
D & Não & Sim & Não \\
E & Não & Sim & Sim \\
F & Não & Não & Sim \\
G & Não & Não & Sim \\
\hline
\end{tabular}

De acordo com a pesquisa, em relação ao deslocamento até a escola, apurados com auxílio do QUADA, $61(60,4 \%)$ crianças iam a pé, 23
$(22,8 \%)$ iam de bicicleta, $15(14,9 \%)$ de carro e 2 (2\%), conforme mostra a Figura 04.

Figura 04 - Percentual em relação aos meios de locomoção utilizados pelos estudantes para chegar até a escola

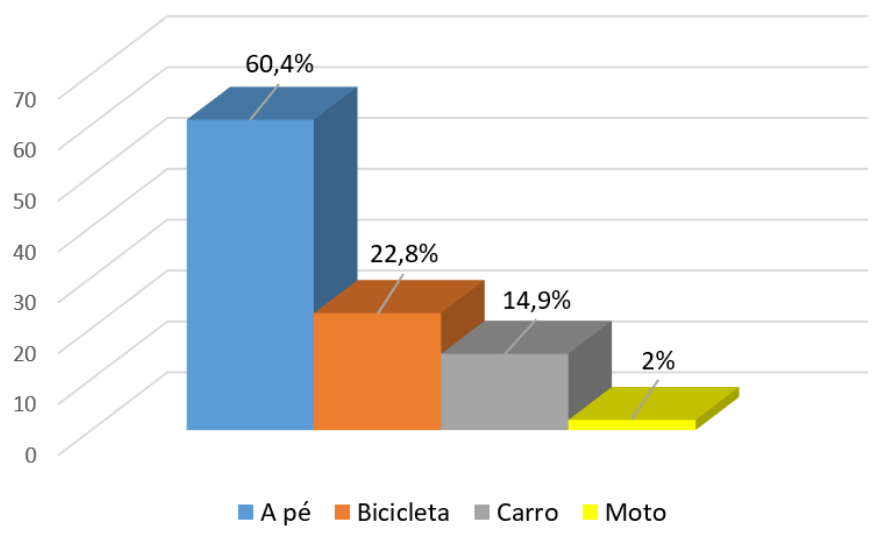


A tabela 04 demonstra os resultados da anamnese sobre o consumo alimentar dos escolares, dividido por categoria de alimentos e refeições. Referente à refeição do café da manhã, as categorias alimentares mais citadas foram laticínios $(63,4 \%)$, pães, bolos ou biscoitos $(53,5 \%)$ e achocolatados, doces, geleias $(30,7 \%)$. O consumo de frutas foi bem baixo (3\%) e o total de crianças que não costumavam realizar essa refeição foi de 28 (27,7\%). No lanche da manhã pôde-se observar que mais da metade das crianças tinham o hábito de consumir a merenda escolar, totalizando 67 pessoas $(66,3 \%)$ e $21(20,8 \%)$ também não tinham o hábito de se alimentar em horário escolar.

Tabela 04 - Consumo alimentar da população assistida, dividido por categorias de alimentos e refeições.

\begin{tabular}{|c|c|c|c|c|c|c|c|c|c|c|c|c|}
\hline \multicolumn{13}{|c|}{ Refeições } \\
\hline \multirow[t]{2}{*}{ Grupos alimentares } & \multicolumn{2}{|c|}{ Café Da Manhã } & \multicolumn{2}{|c|}{$\begin{array}{l}\text { Lanche Da } \\
\text { Manhã }\end{array}$} & \multicolumn{3}{|c|}{ Almoço } & \multicolumn{2}{|l|}{$\begin{array}{l}\text { Lanche Da } \\
\text { Tarde }\end{array}$} & \multicolumn{2}{|l|}{ Jantar } & Ceia \\
\hline & $\mathrm{N}$ & $\%$ & $\mathrm{~N}$ & $\%$ & $\mathrm{~N}$ & $\%$ & $\mathrm{~N}$ & $\%$ & $\mathrm{~N}$ & $\%$ & $\mathrm{~N}$ & $\%$ \\
\hline $\begin{array}{l}\text { Leite e derivados } \\
\text { (leite, queijos, } \\
\text { iogurtes) }\end{array}$ & 64 & 63,4 & 4 & 4 & - & - & 54 & 53,5 & 1 & 1 & 4 & 4 \\
\hline $\begin{array}{l}\text { Carboidratos } \\
\text { complexos (arroz, } \\
\text { macarrão, tubérculos) }\end{array}$ & - & - & - & - & 97 & 96 & 1 & 1 & 80 & 79,2 & - & \\
\hline Frutas & 3 & 3 & 2 & 2 & - & - & 8 & 7,9 & 1 & 1 & 5 & 5 \\
\hline Café ou chá & 36 & 35,6 & - & - & - & - & 34 & 33,7 & - & - & 3 & 3 \\
\hline $\begin{array}{l}\text { Embutidos (presunto, } \\
\text { mortadela, linguiça) }\end{array}$ & 9 & 8,9 & 1 & 1 & - & - & 9 & 8,9 & 2 & 2 & - & - \\
\hline $\begin{array}{l}\text { Doces/açúcar } \\
\text { simples (geleia, } \\
\text { bolacha recheada, } \\
\text { achocolatados) }\end{array}$ & 31 & 30,7 & 4 & 4 & 1 & 1 & 34 & 33,7 & - & - & 4 & 4 \\
\hline Merenda escolar & - & - & 67 & 66,3 & - & - & 1 & 1 & - & - & - & - \\
\hline $\begin{array}{l}\text { Lanches (frituras, pão } \\
\text { de queijo, mini pizza) }\end{array}$ & - & - & 3 & 3 & - & - & 2 & 2 & - & - & - & - \\
\hline $\begin{array}{l}\text { Ultra processados } \\
\text { (salgadinhos) }\end{array}$ & - & - & 1 & 1 & - & - & - & - & - & - & - & - \\
\hline $\begin{array}{l}\text { Bebidas adoçadas } \\
\text { (achocolatados, } \\
\text { refrigerante, suco } \\
\text { industrializado) }\end{array}$ & - & - & 2 & 2 & 31 & 30,7 & 1 & 1 & 17 & 16,8 & - & - \\
\hline Proteína animal & 1 & 1 & - & - & 89 & 88,1 & 1 & 1 & 67 & 66,3 & - & - \\
\hline Leguminosas & - & - & - & - & 84 & 83,2 & 1 & 1 & 53 & 52,5 & - & - \\
\hline
\end{tabular}


Refeições

\begin{tabular}{|c|c|c|c|c|c|c|c|c|c|c|c|c|}
\hline \multirow{2}{*}{ Grupos alimentares } & \multicolumn{2}{|c|}{ Café Da Manhã } & \multicolumn{2}{|c|}{$\begin{array}{c}\text { Lanche Da } \\
\text { Manhã }\end{array}$} & \multicolumn{3}{|c|}{ Almoço } & \multicolumn{2}{|l|}{$\begin{array}{l}\text { Lanche Da } \\
\text { Tarde }\end{array}$} & Jantar & \multicolumn{2}{|c|}{ Ceia } \\
\hline & $\mathrm{N}$ & $\%$ & $\mathrm{~N}$ & $\%$ & $\mathrm{~N}$ & $\%$ & $\mathrm{~N}$ & $\%$ & $\mathrm{~N}$ & $\%$ & $\mathrm{~N}$ & $\%$ \\
\hline Hortaliças e vegetais & - & - & - & - & 47 & 46,5 & 1 & 1 & 25 & 24,8 & - & - \\
\hline Sucos naturais & - & - & - & - & 16 & 15,8 & - & - & 7 & 6,9 & - & - \\
\hline $\begin{array}{l}\text { Carboidratos simples } \\
\text { (pão, bolos, biscoitos) }\end{array}$ & 54 & 53,5 & 12 & 11,9 & - & - & 74 & 73,3 & 7 & 6,9 & - & - \\
\hline Não realiza a refeição & 28 & 27,7 & 21 & 20,8 & 2 & 2 & - & - & 10 & 9,9 & 86 & 85,1 \\
\hline
\end{tabular}

No tocante à refeição do almoço, o grupo de alimentos mais consumido foi do carboidrato e cereais, que abrangeram, na maioria das vezes, alimentos como arroz, polenta, macarrão e tubérculos, estando presente na mesa de 97 (96\%) crianças, $89(88,1 \%)$ das crianças consumiam proteína animal, dentre essas destacam-se as carnes bovinas, suínas, aves, peixes e ovos, e 84 alunos $(83,2 \%)$ que consumiam leguminosas, sendo $100 \%$ das vezes algum tipo de feijão. Foi observado uma alta prevalência do consumo de bebidas adoçadas do tipo refrigerantes e sucos industrializados, atingindo cerca de 31 (30,7\%) indivíduos. Proporções semelhantes foram vistas no jantar, com predominância para o consumo de carboidratos como arroz, macarrão ou tubérculos $(80,79,2 \%)$, feijão $(8,79,2 \%)$ e proteína animal $(67$, $66,3 \%)$. Outros $7(6,9 \%)$ optavam por carboidratos do tipo pães, bolos e biscoitos e $10(9,9 \%)$ crianças que relataram não fazer essa refeição.

No café da tarde, 54 (53,35\%) alunos ingeriam alguma fonte de lacticínios, $73,3 \%(\mathrm{n}=74)$ comiam pães, bolos e biscoitos e apenas $8(7,9 \%)$ crianças relataram ingerir frutas ou sucos naturais.
Seguindo de 34 (33,7\%) que bebiam café ou chá, $9(8,9 \%)$ que consumiam embutidos e $34(33,7)$ que se alimentavam de algum alimento rico em açúcar simples. Referente à ceia, a grande maioria da população estudada $(86,85,1 \%)$ pulava esta refeição, tendo o jantar como último momento de ingesta alimentar.

Porfim, a Tabela 05 demonstra em porcentagem, como estava o consumo alimentar das crianças, tendo como base as porções recomendadas por grupo da Pirâmide Alimentar Infantil Brasileira, coletada pela anamnese alimentar com auxílio do questionário QUADA. A informação que mais chamou a atenção foi que no grupo das frutas, $100 \%$ das crianças consumiam porções abaixo do esperado, e apenas $2 \%$ atingiram a recomendação de consumo de porções de verduras e legumes. Os grupos com maior consumo foram as proteínas animais $(56,4 \%)$ e leguminosas $(41,6 \%)$. É importante salientar que o grupo de óleos e gorduras não foi mensurado, pela falta de informação da adição desse produto durante a cocção. 
Tabela 05 - Porcentagem de crianças em idade escolar que atenderam às recomendações diárias da Pirâmide Aimentar Ifantil Brasileira para o consumo de grupos de alimentos entre refeições principais e lanches, de acordo com a sua anamnese de frequência alimentar em estilo QUADA.

\begin{tabular}{|c|c|c|c|c|}
\hline $\begin{array}{l}\text { Grupo de } \\
\text { alimentos }\end{array}$ & Recomendação* & $\begin{array}{c}\text { Adequado } \\
(\%)\end{array}$ & $\begin{array}{c}\operatorname{Acima}^{\star *} \\
(\%)\end{array}$ & $\begin{array}{c}\text { Abaixo }^{\star * *} \\
(\%)\end{array}$ \\
\hline $\begin{array}{l}\text { Cereais, pães, } \\
\text { tubérculos e } \\
\text { raízes }\end{array}$ & 5 & 23,8 & 11,9 & 65,8 \\
\hline $\begin{array}{l}\text { Verduras e } \\
\text { legumes }\end{array}$ & 3 & 2 & 0 & 98 \\
\hline Frutas & 3 & 0 & 0 & 100 \\
\hline $\begin{array}{l}\text { Leites, queijos } \\
\text { e iogurtes }\end{array}$ & 3 & 16,8 & 1 & 82,2 \\
\hline Carnes e ovos & 2 & 56,4 & 3 & 40,6 \\
\hline Feijões & 1 & 41,6 & 43,6 & 13,9 \\
\hline $\begin{array}{l}\text { Óleos e } \\
\text { gorduras }\end{array}$ & 1 & - & - & - \\
\hline Açúcar e doces & 1 & 33,7 & 34,7 & 31,7 \\
\hline
\end{tabular}

\section{Discussão}

Diversos estudos brasileiros demonstram a classificação nutricional de crianças e adolescentes, tendo em vista a correlação com diversos fatores. Porém, vários destes utilizam como amostra crianças no período pré-escolar, com idade entre 0 e 7 anos de idade, como encontrados nos trabalhos de Silva, et al ${ }^{17}$; Miranda et al. ${ }^{18}$; Fisberg, et al. ${ }^{19}$; Castro et al. ${ }^{20}$ e Garcia et al. ${ }^{21}$. A presente pesquisa, constituiu o aprofundamento da desnutrição na idade escolar, com caráter distinto.

Apenas $1 \%$ dos estudantes apresentaram baixo IMC para idade, tendo baixa prevalência a magreza na população analisada. Com amostra próxima ao estudado nessa pesquisa, Costa et al. ${ }^{22}$, mostram que apenas $2 \%$ dos escolares de 8 a 10 anos de uma escola da rede privada no estado do Piauí apresentaram classificação de magreza segundo IMC/I, seguindo de 73,5\% de eutrofia,
$14,3 \%$ de sobrepeso e $10,2 \%$ de obesidade. Em relação à magreza, foram achados dados similares ao descoberto nesse estudo. Já Gilglioni et al. ${ }^{23}$, estudaram crianças e adolescentes de 6 a 16 anos de idade, em que a maioria dos estudantes $(59,30 \%)$ apresentou peso adequado. Apesar disso, o excesso de peso representou (15,51\%), a obesidade $(14,29 \%)$, o risco para baixo peso $(6,44 \%)$ e baixo peso $(4,46 \%)$.

Segundo o Instituto Brasileiro de Geografia e Estatística (IBGE), o peso das crianças brasileiras vem aumentando nos últimos anos, tendo em vista que no ano de 2009 uma em cada três crianças de 5 a 9 anos estava acima do peso recomendado pela Organização Mundial de Saúde (OMS). O déficit de peso para essa população tinha média nacional de $4 \%$, demonstrando a evolução do país nos últimos anos, principalmente no que se diz respeito ao aumento de renda e diminuição da fome ${ }^{24}$. 
Em contrapartida o Sistema de Vigilância Alimentar e Nutricional, revela que no ano de 2018, município de Joinville, 0,38\% das crianças nesse faixa etária se encontraram em estado de magreza acentuada e 1,96\% com magreza, no total de 1.580 crianças assistidas. Já, no bairro estudado (Aventureiro) não existem crianças de 5 a 10 anos com baixo IMC para idade, no total são colocadas 25 crianças observadas ${ }^{25}$.

O Índice de Desenvolvimento Humano (IDH) da cidade de Joinville/SC era de 0,809 em 2010, colocando a cidade na faixa de Desenvolvimento Humano Muito Alto. $\mathrm{O}$ aspecto que indica maior proporção para o IDHM do município é Longevidade, com índice de 0,889 , continuada de Renda, com índice de 0,795, e de Educação, com índice de $0,749^{26}$.

No que se refere ao Peso/I, diferente do IMC/I, observa-se a menor prevalência de peso elevado, visto que a própria $\mathrm{OMS}^{15}$ dita que a curva de peso para idade não é a melhor ferramenta para classificar sobrepeso/obesidade, devido não levar em consideração a distribuição do peso pela altura das crianças, como feito na curva de IMC/I. Sobretudo, o baixo peso se elevou em comparação com IMC/I.

Entre as crianças, 3\% demostraram baixa estatura para idade, sendo este valor muito próximo do revelado em outros estudos, contudo para população do sul do país. O IBGE através da POF (Pesquisa de Orçamentos Familiares) ${ }^{24}$, mostra que no ano de 2009, o déficit de altura entre as crianças brasileiras de 5 a 9 anos era de 6,8\%, sendo no sul do país $4,7 \%$ para os meninos e $4,0 \%$ para as meninas, a queda desses valores condizem com a queda da desnutrição infantil no país. Ramires et al. ${ }^{27}$ destacaram que entre crianças e adolescentes de 5 a 19 anos, residentes no nordeste brasileiro, em uma amostra total de 860 estudantes, $9,1 \%(\mathrm{n}=78)$ possuíam déficit de estatura. Já no estudo realizado por uma rede de escola privada em âmbito nacional, no ano de 2011/2012 Anjos \& Silveira ${ }^{28}$, detectaram que $1,6 \%$ das meninas e 1,3\% dos meninos tiveram baixa estatura. Assim, as crianças do presente estudo, residentes no sul do país, demostraram percentual ainda mais baixo no déficit de estatura comparado aos estudos da POF 2008/2009 e Ramires et al., porém, referente à pesquisa de Anjos \& Silveira no âmbito de uma escola da rede privada, o percentual encontrado foi superior.

A criança que apresentou IMC abaixo do esperado, também demonstrou baixo peso para idade, porém ficou negada a existência de baixa estatura para a idade. Outras 3 crianças apresentaram apenas déficit negativo no peso para a idade, sem alterações na altura. Apenas um estudante obteve resultado negativo no P/I e E/I, estando ambos abaixo do esperado. Seguido de 2 alunos os quais se destacaram apenas no déficit E/I, sem intercorrências nas curvas de peso e IMC.

Devido à pesquisa não se aprofundar em tempo e quantidade de atividade física, não se define sedentarismo a nenhum estudante. A OMS estabelece a prática de atividade física como prevenção para diversas patologias, inclusive para o sobrepeso e a obesidade ${ }^{29}$. Rivera et al..$^{30}$, em uma amostra de 1.253 alunos entre 7 e 17 anos, identificaram 225 crianças e 947 adolescentes em situação de sedentarismo. Oliveira et al. ${ }^{31}$, mostram que $58,4 \%$ das crianças estudadas tinham como atividade física o deslocamento ativo, resultados semelhantes à atual pesquisa.

A análise dos resultados deste estudo demostra baixa prevalência de desnutrição infantil, tanto em relação ao IMC/I, quando ao P/I. Todavia, ressaltase a baixa adequação no consumo dos principais grupos de alimentos, conforme recomendado pela pirâmide alimentar para idade escolar. Esses dados convencem que a alimentação da grande maioria das crianças se torna pobre em micronutrientes, principalmente pelo baixo consumo de frutas e hortaliças ${ }^{32}$. Além disso, apenas $56,4 \%$ dos escolares mantinham ingestão adequada de proteínas, sendo este um importante fator para evitar a desnutrição energético-proteica ${ }^{33}$.

Outro estudo utilizando o método QUADA, mostrou que, 6,5\% da população estudada possuiu adequação de consumo na categoria de cereais, pães, tubérculos e raízes, em contrapartida de $23,8 \%$ analisado nesse trabalho. Nas demais categorias de alimentos, as crianças desse estudo tiveram menor prevalência de adequação com as quantidades de porções recomendadas ${ }^{34}$. 


\section{Conclusão}

Com este estudo, pôde-se observar uma baixa prevalência de desnutrição em crianças em idade escolar residentes de um município de grande porte, quando da classificação do estado nutricional. Conforme as curvas da $\mathrm{OMS}^{15}$ os déficits no peso e na estatura das crianças foram encontrados em pequena quantidade, assim como verificado em diversos trabalhos recentes. O sobrepeso e a obesidade foram os maiores problemas nutricionais observados na população estudada, fato este correlacionado à transição nutricional.

A alimentação das crianças estudadas tinha como base alimentos como pão, margarina, arroz e feijão, sendo a inadequação no consumo de frutas e hortaliças um dos pontos de maior preocupação. Desta forma, ações de intervenção nutricional são importantes, visando uma educação precoce para prevenção de déficits nutricionais na vida adulta.

Por isso, é importante verificar as carências na qualidade nutricional de crianças residentes em zonas carentes, visto uma vez que a classificação antropométrica do estado nutricional não demonstra a saúde a nível de consumo de macro e micronutrientes. Logo, mais estudos que abordem o consumo alimentar infantil e escolar e seu estado nutricional se fazem necessários.

\section{Referências}

1. Pedroza DF, Silva FA, Melo NLS. Estado nutricional e hábitos alimentares de escolares de Campina Grande, Paraíba, Brasil. Campina Grande. Monografia [Especialização em Enfermagem] - Universidade Estadual da Paraíba, 2017.

2. Araújo LMP. Percepção de perigo e coesão social: impacto dos fatores sociais e comportamentos sedentários no estado nutricional das crianças do município da Lousã. Lousã. Tese [Doutorado em Biologia Humanas] Universidade de Coimbra, 2013.

3. Kucharski, Fernanda Aguiar et al. Perfil de famílias com crianças desnutridas internadas em uma instituição na cidade de Fortaleza-Ceará. Northeast Network Nursing Journal, 2002.

4. Galvão, Maria Aparecida Mendes. Fatores determinantes de desnutrição infantil em crianças de 0 a 05 anos no Cernutri - Boa Vista/Roraima. 2014. 28 f. Monografia (Especialização) - Curso de Enfermagem, Universidade Federal de Santa Catarina, Florianópolis Sc, 20154.
5. Brasil, Presidência da República Casa Civil Subchefia para Assuntos Jurídicos. Lei № 11.346, De 15 De Setembro De 2006. Brasília, Df, Set 2006.

6. Kepple, Anne Walleser; SEGALL-CORRêA, Ana Maria. Conceituando e medindo segurança alimentar e nutricional. Ciência e Saúde Coletiva, Campinas, 2011.

7. Brasil. O que é o CONSEA? 2017.

8. Martins, Cristina. Avaliação do Estado Nutricional e Diagnóstico. Nutroclínica, 2008.

9. Monteiro, Carlos Augusto; CONDE, Wolney Lisboa. Tendência secular da desnutrição e da obesidade na infância na cidade de São Paulo. Revista de Saúde Pública, São Paulo, 2000.

10. Brasil, Ministério da Saúde. Resolução No 466, De 12 De Dezembro De 2012, Brasília, 2012.

11. Direção Geral Da Saúde. Nutrição e Deficiência(s). Lisboa, Portugal: Programa Nacional Para Promoção da Alimentação Saudável, 2015.

12. Yang, Yuchi, LUCAS Betty, FEUCHT Sharon. Nutrition Interventions for Children with Special Health Care Needs. 3 ed.: The Washington State Department of Health; 2010

13. Brasil. Ministério da Saúde. Vigilância alimentar e nutricional: Antropometria: como pesar e medir. Brasília: Ministério da Saúde; 2004 Assis Ma, Benedet J, Kerpel R, Vasconcelos FAG, Di Pietro PF, Kupek E. Validação da terceira versão do Questionário Alimentar do Dia Anterior (QUADA-3) para escolares de 6 a 11 anos. Cad Saúde Pública. 2009;25(8):1816-26.

14. OMS, Organização Mundial da Saúde. Curvas de crescimento da OMS, 2007.

15. SBP, Sociedade Brasileira De Pediatria. Pirâmide alimentar infantil. 2012

16. Silva, Marina Vieira da et al. Acesso à creche e estado nutricional das crianças brasileiras: diferenças regionais, por faixa etária e classe de renda. Revista de Nutrição, 2000 .

17. Miranda, Adriana da Silva et al. Anemia ferropriva e estado nutricional de crianças com idade de 12 a 60 meses do município de Viçosa, MG. Revista de Nutrição, 2003.

18. Fisberg, Regina Mara; MARCHIONI, Dirce Maria Lobo; CARDOSO, Maria Regina Alves. Estado nutricional e fatores associados ao déficit de crescimento de crianças frequentadoras de creches públicas do Município de São Paulo, Brasil. Cadernos de Saúde Pública, 2004.

19. Castro, Teresa Gontijo det al. Caracterização do consumo alimentar, ambiente socioeconômico e estado nutricional de pré-escolares de creches municipais de Viçosa, MG. (Characteristics of dietary intake, socioeconomic environment and nutritional status of preschoolers at public kindergartens). 2005. 
20. Grcia, Mariana Tarricone; GRANADO, Fernanda Serra; CARDOSO, Marly Augusto. Alimentação complementar e estado nutricional de crianças menores de dois anos atendidas no Programa Saúde da Família em Acrelândia, Acre, Amazônia Ocidental Brasileira. Cadernos de Saúde Pública, 2011.

21. Costa, Mayara Carvalho et al. Estado nutricional, práticas alimentares e conhecimentos em nutrição de escolares. Revista de Atenção à Saúde (antiga Rev. Bras. Ciên. Saúde), 2018.

22. Gilglioni, Eduardo Hideo; VARELA FERREIRA, Taiana; BENNEMMAN, Rosi Mari. Estado nutricional dos alunos das escolas da rede de ensino Municipal de Maringá, Estado do Paraná, Brasil. Acta Scientiarum. Health Sciences, 2011.

23. Brasil, IBGE, Portal Brasil. POF 2008-2009: desnutrição cai e peso das crianças brasileiras ultrapassa padrão internacional. 2009.

24. Brasil; Ministério da saúde. Sistema Nacional de Vigilância Alimentar. Brasília, 2018.

25. PNUD, Atlas Do Desenvolvimento Humano do Brasil, 2013.

26. Ramires, Elyssia Karine Nunes Mendonça et al. Estado nutricional de crianças e adolescentes de um município do semiárido do Nordeste brasileiro. Revista Paulista de Pediatria, 2014.

27. Anjos, Luiz Antonio dos; SILVEIRA, Willian Dimas Bezerra da. Estado nutricional dos alunos da Rede Nacional de Ensino de Educação Infantil e Fundamental do Serviço Social do Comércio (Sesc), Brasil, 2012. Ciência \& Saúde Coletiva, 2017.

28. WHO, World Health Organization. Physical activity. Washington; 2003.

29. Rivera, Ivan Romero et al. Atividade física, horas de assistência à TV e composição corporal em crianças e adolescentes. Arq bras cardiol,, 2010.

30. Oliveira, Thiara Castro de et al. Atividade física e sedentarismo em escolares da rede pública e privada de ensino em São Luís. Revista de saúde pública, 2010.

31. Bueno, Aline Lopes; CZEPIELEWSKI, Mauro Antônio. Micronutrientes envolvidos no crescimento. Revista HCPA. Porto Alegre, 2007.
32. Monte, Cristina MG. Desnutrição: um desafio secular à nutrição infantil. J Pediatria, 2000. Assis, Maria Alice Altenburg de et al. Análise qualitativa da dieta de amostra probabilística de escolares de Florianópolis, Santa Catarina, Brasil, com o uso do Questionário Alimentar do Dia Anterior. Cadernos de Saúde Pública, 2010.

\section{Como citar este artigo:}

Chagas JE, Debortoli CC. Prevalência de desnutrição e consumo alimentar em escolares de uma escola municipal de Joinville-SC. Rev. Aten. Saúde. 2019; 17(62): 59-70. 